46 | 2011

Accueil et formation des enfants étrangers en France de la fin du $\mathrm{XIX}^{\mathrm{e}}$ siècle au début de la Deuxième Guerre mondiale

\title{
Parcours de lecture.Enfants de migrants
}

\section{Gérard Vigner}

\section{(2) OpenEdition}

\section{Journals}

Édition électronique

URL : https://journals.openedition.org/dhfles/2111

DOI : $10.4000 /$ dhfles. 2111

ISSN : 2221-4038

\section{Éditeur}

Société Internationale pour l'Histoire du Français Langue Étrangère ou Seconde

Édition imprimée

Date de publication : 1 janvier 2011

Pagination : 161-178

ISSN : 0992-7654

Référence électronique

Gérard Vigner, «Parcours de lecture.Enfants de migrants », Documents pour l'histoire du français langue étrangère ou seconde [En ligne], 46 | 2011, mis en ligne le 31 janvier 2014, consulté le 31 mai 2021. URL : http://journals.openedition.org/dhfles/2111 ; DOI : https://doi.org/10.4000/dhfles.2111

Ce document a été généré automatiquement le 31 mai 2021

(c) SIHFLES 


\title{
Parcours de lecture.Enfants de migrants
}

\author{
Gérard Vigner
}

1 Si la bibliographie concernant les enfants de migrants est plus nettement étoffée s'agissant de la période contemporaine, après la Deuxième Guerre mondiale notamment, celle qui concerne ces mêmes publics pour les périodes antérieures se révèle plus restreinte et doit être recherchée à l'intérieur même d'ouvrages qui traitent de l'histoire de l'immigration dans la période concernée; domaine de recherche, celui de l'histoire de l'enfance et de l'adolescence migrantes dans la première moitié du XX siècle, qui commence à être abordé, de façon encore marginale.

2 Nous proposons ici un parcours de lecture, qui ne prétend nullement à l'exhaustivité, mais qui permettra de présenter un certain nombre d'éléments de contexte susceptibles d'éclairer ce que fut la situation des enfants de migrants, pris eux-mêmes dans l'histoire de leur famille.

\section{Un cadre historique général}

Gérard Noiriel. Le Creuset français. Histoire de l'immigration, $\mathrm{XIX}^{e}-\mathrm{XX}^{e}$ siècles, Paris, Seuil, 1988, $437 \mathrm{p}$.

4 Ouvrage de référence, que l'on peut considérer comme un classique en la matière et qui a posé les grands repères de ce qu'a pu être une histoire de l'immigration. L'auteur souhaite montrer que les approches sociologiques, en synchronie, ne sauraient suffire à rendre compte d'une composante, longtemps négligée, de la vie française, qui a besoin $\mathrm{du}$ recul historique pour trouver sa pleine signification.

5 Marie-Claude Blanc-Chaléard. Histoire de l'immigration, Paris, La Découverte, 2001, $120 \mathrm{p}$.

6 Ouvrage qui dans un nombre limité de pages offre une très claire synthèse de l'histoire de l'immigration en France, pays qui offre cette particularité quasi unique en Europe d'être « un pays d'immigrants dans un continent d'émigrants ». L'ouvrage s'organise en 
un découpage chronologique qui nous conduit de la guerre de 1914 à aujourd'hui, selon une histoire dans laquelle la place de l'étranger a toujours été difficilement reconnue, le souci d'intégration à la française n'excluant pas à certains moments de fortes poussées xénophobes.

7 Janine Ponty. L'immigration dans les textes. France, 1789-2002, Paris, Belin, 2004, 415 p.

8 Deux cents documents sont ici rassemblés par Janine Ponty. L'exil, la nationalité, l'intégration, l'identité, l'école, la deuxième génération, sont les fils qui réunissent ces documents, soigneusement inscrits dans leur contexte et qui de la sorte dessinent une autre histoire de l'immigration, dans laquelle racisme et xénophobie apparaissent à bien des moments. Un instrument de travail utile pour toutes celles et tous ceux qui veulent examiner de près, ce que furent à un moment donné les politiques mises en œuvre à l'égard des populations immigrées.

Philippe Rygiel. Le temps des migrations blanches. Migrer en Occident (1840-1940), Montreuil, Aux lieux d'être, 2007, 227 p.

Dans un ouvrage à visée largement synthétique, l'auteur retrace un très profond et très puissant mouvement qui sur un peu plus d'un siècle a mis en branle, dans une poussée générale vers l'ouest, des dizaines de millions d'individus, d'origine européenne, depuis la Russie jusqu'aux différents États du continent américain (on estime ainsi à environ 55 millions le nombre de personnes qui ont franchi l'Atlantique pour se rendre au Canada, aux États-Unis et en Argentine). Mais aussi une poussée vers l'Europe de l'Ouest de migrations issues d'Europe centrale et orientale et d'Europe du Sud. Les traités issus de la Première Guerre mondiale seront ainsi à l'origine de déplacements importants de population. L'intérêt de l'ouvrage réside dans la vision d'ensemble qu'il propose d'un phénomène dont on n'a pas toujours mesuré l'importance de l'impact dans l'histoire de l'Europe.

Marie D. Lewis. Les frontières de la République. Immigration et limites de l'universalisme en France (1918-1940), Marseille, Agone, 2010, 425 p.

Sous un titre apparemment très général, l'auteur en fait aborde de plus près ce que fut la condition des immigrants dans deux villes de province, Lyon et Marseille. En effet, si le gouvernement définit bien une politique générale en matière d'immigration, sa mise en œuvre est le fait des administrations locales qui interprètent les directives nationales en fonction des particularités locales. Lyon, après la Grande Guerre connaît une seconde industrialisation et de forts besoins de main-d'œuvre; Marseille, par ses activités portuaires a besoin d'une main-d'œuvre particulièrement flexible. De cette étude extrêmement fouillée et qui s'appuie sur des sources délaissées, il ressort que les migrants sont dans une situation de grande vulnérabilité juridique, pris dans des réseaux de circulaires et de règlements, souvent contradictoires, rédigés au gré de l'évolution des situations économiques et politiques (crise économique de 1929, approche de la guerre) et des réactions des hommes politiques et des représentants syndicaux. Populations migrantes aux statuts variés: ressortissants de nations signataires de traités, telle l'Italie par exemple; migrants issus de nations non contractantes, Russie ${ }^{1}$, Arménie, réfugiés fuyant le nazisme, migrants venus d'Afrique du Nord, sujets d'empire et non-citoyens. Disparités des droits sociaux, des droits au travail, entre migrants aux statuts spécifiques, entre migrants et travailleurs nationaux qui ne manquent pas de marquer leur hostilité à cette population supposée menacer l'emploi et les droits qui y sont associés. Les responsables syndicaux ne manquent pas 
dans certains cas de se faire l'écho de telles plaintes. Disparité aussi des politiques conduites à Lyon et à Marseille encore. Universalisme républicain mis à mal par une migration de masse qui s'inscrit dans des dynamiques contradictoires d'exclusion et d'inclusion. Mais ouvrage qui montre aussi qu'il n'a certainement jamais existé d'âge d'or d'une intégration républicaine des étrangers.

\section{Histoire de la jeunesse, histoire de l'enfance}

13 La jeunesse fait partie de ces catégories sociologiques aux frontières incertaines (quand cesse-t-on d'être jeune par exemple? les bornes de la jeunesse sont-elles placées de façon identique dans tous les pays? pour les garçons comme pour les filles?) que les historiens n'ont construit en objet d'étude que tout récemment. Certes Maurice Crubellier, dans la fin des années 70, avait publié une vaste synthèse, L'enfance et la jeunesse dans la société française, 1800-1950,Armand Colin, 1979, et depuis les travaux se sont plus largement développés. Le projet franco-italien de portée plus vaste encore de Giovanni Levi \& Jean-Claude Schmitt (dir.),Histoire des jeunes en Occident, PUF, 1996, en deux forts tomes. Agnès Thiercé encore avec son Histoire de l'adolescence 1850-1914, Armand Colin, 1999, qui explore cette catégorie plus instable encore qu'est celle de l'adolescence.

14 S'intéresser aux enfants de migrants ne peut donc se faire sans prendre en considération ce que fut la jeunesse et son traitement social à un moment donné de l'histoire de la France. En effet, par l'école, par des loisirs partagés, par la diffusion et l'appropriation, plus ou moins prononcée, de normes de socialisation, les enfants de migrants sont au premier rang de ces processus complexes d'intégration, vécus et mis en œuvre différemment de ceux présents chez les adultes. Connaître cette histoire de la jeunesse permet donc de mieux situer, d'un point de vue à la fois historique et sociologique, la dynamique et les trajectoires d'intégration. En effet, un enfant, un adolescent issu de familles migrantes est aussi un jeune au sens le plus général du terme et adhère pour partie ou totalement, selon les individus, aux normes et valeurs du pays d'accueil. Et l'on sait combien dans les familles migrantes les tensions ont été fortes, ou le sont encore, entre adultes et enfants. Connaître ce que fut l'histoire de cette jeunesse peut éclairer sur quelques points ce qu'a pu être à un moment donné le fait d'être un jeune venu d'ailleurs.

Deux ouvrages méritent cependant d'être cités :

Ludivine Bantigny et Ivan Jablonka (dir.). Jeunesse oblige. Histoire des jeunes en France XIX ${ }^{e}$-XXe siècles, Paris, PUF, 2009, 307 p.

L'ouvrage réunit dix-neuf communications présentées lors d'un séminaire tenu au Centre d'histoire de Sciences Po à partir de 2005. Peu de contributions cependant consacrées aux jeunes dans la première moitié $d u \mathrm{XX}^{\mathrm{e}}$ siècle. Parcours très riche, inégalement distribué dans sa thématique et sa chronologie et dans lequel sont abordés des thèmes tels que celui des identités juvéniles successives, la question de la jeunesse délinquante ou déviante selon les dénominations de certains auteurs, l'histoire du service militaire qui pendant longtemps fut vécu tout à la fois comme une épreuve personnelle et un rite de passage indispensable, les modes de vie et les cultures juvéniles, rock et yéyés, l'âge des expériences sexuelles, bref un tableau qui, s'il n'est pas complet, permet de penser différemment une classe d'âge dont les modes de perception et de délimitation ont toujours posé problème. La question des jeunes issus 
de l'immigration n'y est abordée qu'à la marge. Mais l'ouvrage pose ainsi des jalons qui peuvent être repris en vue de développer une meilleure connaissance de ces publics largement ignorés dans la première moitié $\mathrm{du} \mathrm{XX}^{\mathrm{e}}$ siècle.

Ivan Jablonka. Les enfants de la République. L'intégration des jeunes de 1789 à nos jours, Paris, Seuil, 2010, 357 p.

19 L'ouvrage d'Ivan Jablonka est consacré à ces figures de l'altérité juvénile que furent, selon les époques et il est intéressant d'en apprécier la diversité des dénominations, les bâtards, dévoyés, les enfants abandonnés et les délinquants en tous genres, « enfants de la loi », c'est-à-dire soumis à l'action d'un État qui tout à la fois les stigmatise et veut les sauver. Le parcours, très soigneusement tracé, depuis les politiques de la Révolution jusqu'aux actions conduites aujourd'hui en direction des «jeunes des cités », tente de répondre à cette question : «Et si les jeunes des cités n'avaient qu'un lien indirect avec les banlieues, l'immigration et le colonialisme ? Et s'ils étaient le produit et les héritiers

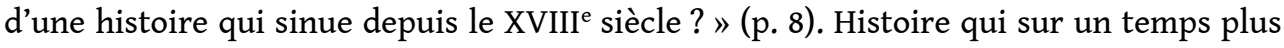
long permet de sortir d'un présent saturé de considérations ethniques dans l'analyse du phénomène, au profit d'une politique générale en direction des jeunes qui puise au plus profond de conceptions régénératrices fort anciennes, le jeune étant réhabilité moralement et physiquement parce qu'éloigné de ses origines imparfaites (famille, milieu social). Ouvrage qui étudie les différentes modalités de mise en œuvre, selon les époques (placement de l'enfant en milieu familial rural, colonies agricoles, placement en milieu familial, associés à l'évolution incontestable d'une législation qui aboutira à l'ordonnance de 1945 sur l'enfance délinquante). La quatrième partie, intitulée "La francisation des minorités postcoloniales ", examine les politiques engagées dans l'époque contemporaine en direction d'enfants et de jeunes dont l'appartenance initiale à la France de la République n'était nullement évidente. Utopie intégratrice mise souvent à mal, d'un modèle républicain bien problématique. Ivan Jablonka conclut ainsi sur ce qui pourrait passer pour une situation paradoxale: "Indissociablement universaliste et chauvin, égalitaire et discriminatoire, abstrait et sexiste, le modèle français d'intégration n'est donc pas en crise ; au contraire, élevé en religion d'État, il se porte à merveille » (p. 301).

\section{Immigrations singulières}

\subsection{La migration flamande dans le nord de la France}

(Références bibliographiques proposées par Walter Kusters, université de LeuvenCourtrai)

\subsection{La migration polonaise}

Janine Ponty, professeur émérite des universités, a consacré toute sa carrière à l'étude de la migration polonaise en France et à ce titre a publié de très nombreux travaux. Nous ne ferons qu'en signaler ici les plus significatifs.

Janine Ponty. «Une intégration difficile : les Polonais en France dans le premier vingtième siècle ", $X X^{e}$ siècle, 1985, vol. 7, p. 51-59.

Etude qui rappelle les conditions, difficiles, dans lesquelles les Polonais s'acclimatèrent en France, et sur le malentendu qui pendant longtemps subsista entre Français et 
Polonais. Janine Ponty, dans cette analyse brève mais fort bien documentée, rappelle que: «l'immense majorité des Français n'a jamais rien compris, dans les années 1920-1930, au comportement des immigrés polonais : ni les autorités civiles, obsédées par la crainte de la constitution d'un État dans l'état, ni la hiérarchie catholique, soucieuse d'unité et avide de faire rentrer les fidèles polonais dans le cadre habituel des paroisses, ni les maîtres d'école persuadés de la supériorité de la culture française et du passé prestigieux de la France. Les Polonais, comme les autres petits étrangers et comme les enfants scolarisés dans les colonies ou les protectorats, devraient se mettre en tête que les Gaulois sont leurs ancêtres. Mais ils s'y refusent parce que leurs parents, les moniteurs scolaires et les aumôniers leurs parlent d'autres ancêtres. Ils apprenant bien en classe, parlent vite le français sans accent mais se veulent Polonais. » Vécus et représentations de la génération née entre 1925 et 1940, Paris, L'Harmattan, 1999, 223 p. 

par le groupe de travail «Péninsule ibérique » du Centre d'histoire de l'Europe du XX siècle de la Fondation nationale des Sciences politiques. Ancien, au moins relativement, mais important par la connaissance qu'il apporte sur la génération des enfants de la guerre civile espagnole. Autant en effet abondent les ouvrages consacrés à la guerre civile espagnole, de même que ceux consacrés à l'exil des républicains espagnols, autant les travaux qui portent sur le sort des enfants pris dans la tourmente de la guerre civile, puis dans celle de la Deuxième Guerre mondiale sont moins nombreux. L'ouvrage rassemble des communications de chercheurs français et espagnols et apporte des éclairages procédant de points de vue variés : sort des enfants basques et catalans, envois d'enfants dans des colonies de vacances sur le territoire français, difficultés liées à la fréquentation de l'école, enfants accueillis en URSS puis retrouvés par les troupes allemandes et renvoyés en Espagne, intégration des enfants exilés et transmission d'une mémoire, engagement politique des adultes issus de cette génération, écho enfin de cette expérience et de cette mémoire dans un certain nombre films et d'ouvrages littéraires.

Geneviève Dreyfus-Armand. L'exil des Républicains espagnols en France. De la guerre civile à la mort de Franco, Paris, Albin Michel, 1999, 475 p.

31

L'immigration espagnole en France est bien antérieure à la guerre civile d'Espagne. Dès le $\mathrm{XIX}^{\mathrm{e}}$ siècle une colonie espagnole est présente en France, constituée d'opposants politiques, mais comprenant aussi de nombreux ouvriers agricoles. En 1901 on décompte environ 80000 émigrants péninsulaires en France. En 1931, ils sont un peu plus de 350000 (chiffres tirés de Marie-Catherine TalvikkiChanfreau, «Espagnols en territoire français de 1813 à 1971 : circuits ou intégrations d'exilés et d'émigrés ", Cahiers du MIMMOC, $\mathrm{n}^{\circ}$ 1, fév. 2006). Mais la guerre civile d'Espagne va marquer un tournant particulièrement dramatique dans une histoire que retrace Geneviève Dreyfus-Armand. Un exode sans équivalent comme le signale d'emblée l'auteur, la Retirada (la retraite), qui se produit après la chute de la Catalogne en janvier 1939, puisque environ un demi-million de personnes se réfugient en France dans le premier trimestre de 1939. Dans une France méfiante, parcourue de courants xénophobes et débordée devant un afflux aussi massif. L'ouvrage suit de façon attentive le parcours d'exil de ces réfugiés espagnols, depuis l'arrivée en France et le passage quasiment sans transition d'une guerre à l'autre, la place très difficilement concédée aux républicains espagnols par la France de Vichy, l'engagement de certains dans la Résistance, puis les années d'après-guerre qui oscillent entre espérance de voir disparaître le régime franquiste et désillusion devant la pérennisation de l'Espagne franquiste. Un exil qui va connaître des temps difficiles. Soumis à l'épreuve d'une durée qui érode les engagements, la vie quotidienne est là avec ses contraintes, qui voit arriver de nouveaux émigrés espagnols dans les années 50 et 60 , aux effets des nombreuses naturalisations qui modifient le profil d'engagement des familles, l'exil de provisoire devient progressivement définitif. Geneviève Dreyfus-Armand retrace dans le détail les activités militantes, publications, mais aussi clivages, et éloignement progressif d'une Espagne réelle souvent vécue dans le souvenir d'une référence historique, celle de la guerre civile, de plus en plus distante. L'auteur retrace aussi les difficiles chemins d'une intégration qui a permis cependant de modifier les représentations que l'opinion française se faisait de l'Espagnol. L'ouvrier agricole misérable du début du siècle laissa place à un résistant courageux, à des personnes, travailleurs, artistes, intellectuels, 
ayant pu trouver un ancrage profond dans la société française. Le sort des enfants n'est pas évoqué ici, mais quelque part en creux définit quelques grandes étapes, enfants pris dans le traumatisme initial de l'exil avec des familles dont la préoccupation première est d'assurer la survie matérielle, puis une intégration progressive dont l'école sera le vecteur principal.

\subsection{La migration arménienne}

(Références bibliographiques proposées par Jean-Sébastien Gauthier)

\subsection{Les migrations juives d'Europe centrale}

Le sort tragique qui a été celui d'un certain nombre d'enfants juifs vivant en France, raflés, déportés et morts à Auschwitz durant l'Occupation, a donné lieu à la publication de nombreux ouvrages. D'autres enfants ont pu être envoyés dans le Massif Central, ce qui leur a permis d'échapper à un sort tragique: cf. Katy Hazan, Serge Klarsfeld \& Cecilia Walters, Le sauvetage des enfants juifs pendant l'Occupation, dans les maisons de l'OSE, 1938-1945 - L'Euvre de secours aux enfants, édition bilingue français-anglais, Paris, Somogy, 2008.

Nombreuses sont les études qui leur sont consacrées, toutes intéressantes à des degrés divers, mais qui nous situent bien au-delà, ne serait-ce que par l'intensité dramatique, de la problématique de notre rencontre. Pour autant, on peut se reporter à un certain nombre de publications qui définissent le cadre dans lequel les enfants réfugiés d'Europe centrale avec leurs familles ont pu être accueillis.

Notamment, dans le $\mathrm{n}^{\circ} 1$ de la revue Les Cahiers de la Shoah, deux articles qui rendent compte des difficultés d'accueil: Anne Grynberg, «L'accueil des réfugiés d'Europe centrale en France (1933-1939) », 1994, les Éditions Liana Levi; et le rappel d'une conférence méconnue, la conférence d'Évian, qui s'est tenue en juillet 1938, et dans laquelle un certain nombre de pays occidentaux, États-Unis, Grande-Bretagne, France et vingt-six autres pays d'Europe se réunissent pour examiner les moyens de faciliter l'émigration des réfugiés politiques en provenance d'Autriche et d'Allemagne, Catherine Nicault, «L'abandon des Juifs avant la Shoah: la France et la conférence d'Évian ". Conférence peu connue, disions nous, mais qui d'une certaine manière entérina le sort des juifs d'Europe centrale présents en Europe et qui ne purent bénéficier de l'aide espérée. Leur sort tragique y fut d'une certaine manière scellé.

\subsection{La migration italienne}

La migration italienne ne peut bien évidemment être omise dans la mesure où elle fut certainement une des plus importantes d'Europe par le nombre de personnes émigrant à partir d'un pays donné (on estime en effet à environ 26 millions le nombre de personnes qui ont quitté l'Italie entre 1870 et 1950, cf. Caroline Douki, « 26 millions d'émigrés ", Les Collections de l'Histoire, $\mathrm{n}^{\circ}$ 50, janvier-mars 2011) et où elle représenta, pour la France, jusqu'au début de la Deuxième Guerre mondiale, la première population immigrée (un peu plus de 2 millions de personnes dans la France métropolitaine, sans oublier qu'est établie à la même époque une importante colonie italienne dans la Tunisie du protectorat, environ 100000 personnes aux alentours de 1936). 
Monique Rouch et Carmela Martone (dir.). Sur les pas des Italiens en Aquitaine au vingtième siècle, en signalant plus particulièrement la communication de Patrick Veglia, "L'école, adaptation et intégration", Maison des sciences de l'homme d'Aquitaine, 1997.

Colloque qui étudie sous différents aspects l'histoire des Italiens dans le Sud-Ouest. On signalera notamment une communication de Carmela Martone, "Les associations fascistes italiennes dans le Sud-Ouest » (doposcuala, casa degli italiani) qui fait apparaître l'importance de l'effort engagé par les autorités italiennes pour conserver un certain contrôle des populations émigrées. Des fasci sont implantés dans le cadre des circonscriptions consulaires et les consuls italiens joueront un rôle important dans l'organisation et l'animation d'une politique d'encadrement. Gianfausto Rosoli dans «Les missionnaires italiens dans le Sud-Ouest rural français » retrouve le thème bien connu dans les histoires de l'émigration du rôle joué par le clergé dans la conservation d'une identité nationale (rôle que l'on peut retrouver dans les émigrations irlandaises ou polonaises en différents endroits $d u$ monde). Tensions entre le modèle assimilationniste français et les résistances nationales au travers notamment d'une église qui dans certains cas pouvait adopter la langue italienne pour le prêche dominical. Mais l'on accordera une attention plus particulière à la communication de Patrick Veglia, «L'école, adaptation-intégration », qui met en évidence l'importance du rôle joué par l'école comment agent d'adaptation. Les enfants italiens représentent environ $10 \%$ des effectifs, mais dans certains endroits cette proportion peut se révéler plus élevée, les cursus scolaires des petits Italiens, fréquentation, assiduité, s'organisent selon des logiques proches de celles des petits Français ${ }^{2}$.

Laure Teulières. Immigrés d'Italie et paysans de France, 1920-1944, Toulouse, Presses universitaires du Mirail, 2002, 263 p.

Le fait migratoire est le plus souvent associé à l'installation de populations étrangères dans des régions industrielles (mines, industrie sidérurgique, construction automobile, etc.), ce qui est vérifié dans un très grand nombre de cas. Mais c'est oublier qu'il y eut aussi une migration en zone rurale, installation de paysans belges en Picardie et en Normandie à la même époque, soit pour porter aide aux exploitants agricoles à certains moments de l'année (moissons, vendanges), soit pour s'installer dans des fermes que la guerre a en partie vidées de leurs habitants, ce qui fut le cas du Sud-Ouest de la France. Des populations italiennes s'y installèrent, environ 80000 personnes, dès 1920 dans le cadre d'accords divers signées entre le gouvernement italien et les autorités françaises. Laure Teulières, maître de conférences à l'université du Mirail à Toulouse, a consacré cet ouvrage à l'histoire, jusqu'à présent peu connue, de ces populations italiennes, venues pour l'essentiel de l'Italie du nord, installées dans le Sud-Ouest. La partie de cette étude qui nous intéressera le plus, mais toutes sont intéressantes, la troisième, rend compte de l'importance de l'effort de maillage entrepris par les institutions italiennes, État et Église, pour encadrer des populations dont on souhaite préserver l'italianité. Les consulats italiens tenteront d'instaurer le jeudi des cours à destination des enfants scolarisés dans les écoles françaises dispensés par des institutrices italiennes, l'objectif de ces cours étant de préserver tout à la fois l'identité culturelle des élèves et aussi leur allégeance au pouvoir fasciste. En cette fin des années 30, devant l'aggravation des tensions internationales, toute affirmation identitaire devient difficile tolérable devant une opinion publique française qui confond expression de l'italianité et engagement fasciste. Ainsi l'agence consulaire italienne demande en 
février 1937 à officialiser un enseignement du jeudi. Les autorités françaises s'y opposeront, l'État italien, État fasciste, apparaissant de plus en plus comme un État potentiellement ennemi de la France. Le préfet du Gers fera fermer plusieurs cours. Ce qui ne manqua pas, à partir de 1938, de placer les populations italiennes dans une situation difficile par rapport à une opinion publique française qui ne manquait pas de les regarder avec de plus en plus de suspicion.

\section{Marie-Claude Blanc-Chaléard. Les Italiens dans l'Est parisien. Une histoire} d'intégration (1860-1960), Rome, École française de Rome, 2000, 803 p.

Ouvrage imposant par son volume et sa portée qui retrace ce que furent les lieux et l'histoire de la migration italienne dans l'Est parisien sur plus d'un siècle. Époque essentielle qui vit l'immigration italienne passer d'une immigration de musiciens des rues, de colporteurs vendeurs de statuettes (les figuristes lucquois par exemple dont parle Caroline Douki dans son étude «Le territoire économique d'une région d'émigration : campagnes et montagnes lucquoises, du milieu du XIX ${ }^{e}$ siècle à 1914 ", Revue d'histoire moderne et contemporaine, 2001/2, $\mathrm{n}^{\circ} 48-2$,), passage d'une immigration mobile à une immigration massive qui va accompagner le mouvement général d'industrialisation de la France. Migration qui est ici approchée à partir tout d'abord des quartiers de Sainte-Marguerite et de Charonne, dans la proximité du Faubourg Saint-Antoine, jusqu'à Montreuil et Nogent-sur-Marne, une population pour l'essentiel originaire du nord de la péninsule (comme d'ailleurs une grande partie de l'immigration italienne en France, les Italiens du sud s'étant plutôt dirigés vers les États-Unis). On n'oubliera pas en effet que la gare de Lyon à Paris a été le point de débarquement de nombreux immigrés italiens et que pour cette raison l'installation dans l'est de Paris a pu être privilégiée. Espaces de transition dans un processus lent, mais très puissant, d'assimilation. Immigration dans laquelle se mêle, comme dans d'autres régions de France, une immigration économique mais aussi une immigration politique, moins importante en nombre, mais très présente, constituée de groupes antifascistes. Ouvriers du bois, maçons, représentent l'essentiel de cette main d'œuvre.

À chaque moment de cette histoire correspondent des modes d'intégration dans ce que l'auteur nomme le creuset parisien pour se traduire dans les années 50 par un essaimage progressif dans l'espace parisien et banlieusard, dans les cités nouvelles comme dans les pavillons. Le rôle des femmes, et des femmes commerçantes, y est évoqué, de même que l'importance prise par les mariages mixtes dans les processus d'intégration.

On ne manquera pas au passage, remarque purement anecdotique, mais intéressante quant à la genèse des facteurs identitaires culturels, de signaler le rôle des immigrants italiens dans ce qui allait devenir un emblème particulièrement français des cultures populaires, l'accordéon et le bal musette. En effet, jusque vers la fin du XIX ${ }^{\mathrm{e}}$ siècle, les Auvergnats de Paris avaient l'habitude, le dimanche, du côté de la Bastille, de se retrouver dans des bals musettes, c'est-à-dire des bals dans lesquels on dansait au son de la musette, petit instrument à vent, au son aigrelet. Mais des ouvriers italiens amènent avec eux un instrument nouveau, d'origine autrichienne, l'accordéon, instrument doté d'une gamme chromatique infiniment plus étendue et d'une belle présence sonore. Le combat fut rude entre la musette auvergnate et ce nouvel instrument venu de l'étranger, apporté par des étrangers; on imagine la nature des arguments échangés à ce moment-là. Mais l'accordéon l'emporta et désormais triompha dans tous les bals musettes (le nom fut conservé) qui fleurirent autour de la 
Bastille, dont le fameux Balajo, rue de Lappe. Comme quoi les marqueurs de l'identité nationale les plus spécifiques peuvent venir de l'étranger³.

Marie-Claude Blanc-Chaléard, Eric Vial, Bénédicte Deschamps (dir.). Les Petites Italies dans le monde,Presses universitaires de Rennes, 2007, $444 \mathrm{p}$.

On sait, nous l'avons déjà signalé, l'importance de la migration italienne. La France ne fut qu'une destination parmi d'autres. Géographie et histoire des lieux d'implantation en différents endroits du monde, Amérique du Nord et du Sud, Europe, Australie, dans les villes, dans certains quartiers tel Little Italy à New-York, mais aussi à la campagne, Laure Teulières revient sur la situation des Italiens du Sud-Ouest. Comment une identité italienne de l'étranger a-t-elle pu se forger dans la diversité des lieux d'installation?

\section{BIBLIOGRAPHIE}

\section{Géographie :}

Lentacker, Firmin (1974). La frontière franco-belge. Étude géogra-phique des effets d'une frontière internationale sur la vie de relations, Lille : Morel \& Corduant.

\section{Démographie :}

Dupâquier, Jacques (1991). « La contribution des Belges à la formation de la population française (1851-1940). Étude quantitative ». In Société Belge de Démographie (éd.), Historiens et populations : liber amicorum Étienne Hélin. Louvain-la-Neuve, 331-347.

Pétillon, Chantal (2006). La population de Roubaix. Industrialisation, démographie et société (1750-1880), Villeneuve d'Ascq : Presses universitaires du Septentrion.

Pétillon, Chantal (2006). « Roubaix, une ville industrielle nourrie d'immigration belge ». In David Michel, Bruno Duriez, Rémi Lefebvre \& Georges Voix, Roubaix: cinquante ans de transformations urbaines et de mutations sociales, Villeneuve d'Ascq : Presses universitaires du Septentrion, 23-39.

\section{Linguistique :}

Declercq, Elien \& D'hulst, Lieven (2010). « The fate of a migrant language in Northern France (1880-1914): Flemish in song repertoire », Journal of Multilingualism, 7, 225-268.

Landrecies, Jacques. (2001). « Une configuration inédite : la triangulaire français-flamand-picard à Roubaix au début du XX $\mathrm{X}^{\mathrm{e}}$ siècle », Langage et Société, 97, 27-68.

Pooley, Tim (2006). « The linguistic assimilation of Flemish immigrants in Lille (1800-1914) », French Language Studies, 16, 207-233. 


\section{Syndicalisme, vie industrielle :}

Couton, Philippe (2003). « Ethnic Institutions Reconsidered: The Case of Flemish Workers in 19th Century France », Journal of Historical Sociology, 16, 80-110.

Strikwerda, Carl (1998). « France and the Belgian Immigration of the Nineteenth Century ». In Camille Guerin-Gonzales \& Carl Strikwerda (eds.), ThePolitics of Immigrant Workers. Labor Activism and Migration in the WorldEconomy since 1830. New York : Holmes \& Meier.

\section{Ouvrages plus ou moins 'généraux' :}

David, Michel, Guillemin, Alain \& Waret, Philippe (1995). L'intégration des Belges à Roubaix. Rapport de synthèse (Les Cahiers de Roubaix 3), Roubaix : Lire à Roubaix.

Morelli, Anne (éd.) (1998). Les émigrants belges. Réfugiés de guerre, émigrés économiques, réfugiés politiques ayant quitté nos régions du XVİ̀me siècle à nos jours, Bruxelles : Éditions Vie Ouvrière.

Popelier, Jean-Pierre (2003). L'immigration oubliée. L'histoire des Belges en France, Lille :La Voix du Nord.

Bardakdjian, Geneviève. La communauté arménienne de Décines, 1925-1971, mémoire de maîtrise d'histoire sous la direction de Gilbert Garrier, Lyon, Faculté des lettres et sciences humaines de Lyon, 1972, 132, $10 \mathrm{p}$.

Belmonte, Lydie (2004). La Petite Arménie. Histoire de la communauté arménienne à Marseille, Éditions Jeanne Laffitte, $224 \mathrm{p}$.

Boghossian, Stephan (2009). La communauté arménienne de Marseille - Quatre siècles de son histoire, Graveurs de Mémoire, Paris : L'Harmattan, 330 p.

Boudjikanian-Keuroghlian, Aïda (1978). Les Arméniens dans la région Rhône-Alpes, Lyon : revue de Géographie de Lyon.

Demma, Christian (1974). La colonie arménienne de Valence 1923-1973, mémoire de maîtrise d'histoire sous la direction d'Yves Lequin, Faculté des lettres et sciences humaines de Lyon, $160 \mathrm{p}$. Der Alexanian, Jacques (1992). Les héritiers du pays oublié - Arménie, Arménies 1922/1987, Paris : Editions Robert Laffont, $476 \mathrm{p}$.

Dupuis, Lucien. « Familles arméniennes à Romans ", Etudes drômoises, octobre 2004, n 19, p. 31-34.

Garagnon, P. (1956). « La colonie arménienne de Valence-sur-Rhône », Revue de géographie alpine, Fascicule III, Grenoble.

Gauthier, Jean-Sébastien. « Les 'écoles arméniennes', Madier-de-Montjau et du Palais », Revue drômoise, $\mathrm{n}^{\circ}$ 515, mars 2005.

- « La scolarisation des enfants arméniens de Valence des années 20 à nos jours », Actes du colloque de Valence «Institutions et immigration », 2 et 3 février 2007, Revue drômoise «Institutions et immigration - Approches locale et nationale - XIX ${ }^{\mathrm{e}}-\mathrm{XX}^{\mathrm{e}}$ siècles », $\mathrm{n}^{\circ} 534$, décembre 2009.

Huard, Jean-Luc. L'arrivée des Arméniens dans la Drôme des années 1920 à 1940, Revue drômoise, 2002, fascicule 505.

- « La présence arménienne dans l'entre-deux-guerres dans la région Rhône-Alpes », Hommes et migrations, janvier-février 2007, n 1265. 
Jarnbodjian, Daniel. «L'arrivée des Arméniens en Ardèche, une enquête », Mémoire d'Ardèche et Temps présent, 1999, nº4.

Kévorkian, Raymond H. \& Paboudjian, Paul B. (1992). Les Arméniens dans l'empire ottoman à la veille du génocide,Paris : Les éditions d'Arts et d'Histoire, ARHIS, 603 p.

Kévorkian, Raymond H. \& Tachjian, Vahé(dir.) (2006). Un siècle d'histoire de l'Union Générale Arménienne de Bienfaisance, volume 1 (1906-1940), Conseil Central, Le Caire - Paris - New York, 268

p.

Temime, Émile (2007). Histoire des migrations à Marseille, T3 : Le cosmopolitisme de l'entredeux-guerres (1919/1945), Éditions Jeanne Laffitte, 188 p.

\section{NOTES}

1. L'exil russe en France bénéficia cependant de l'appui de l'office central des réfugiés russes, fondé en 1924, qui prit le relais de l'ancienne ambassade de la Russie disparue. On compte à peu près 80000 Russes accueillis en France. Sur cette question, voir l'ouvrage de Catherine Gousseff, L'exil russe. La fabrique du réfugié apatride, Paris, CNRS, 2008, 336 p. On pourra aussi se reporter à un autre ouvrage du même auteur, Catherine Groussef et Anna Sossinskaïan, éds., Les enfants de l'exil. Récits d'écoliers après la Révolution de 1917, Bayard, 2005, ouvrage qui rassemble 56 récits recueillis auprès d'enfants russes, exilés après la Révolution et la guerre civile, et placés dans des lycées russes en exil, orphelins regroupés dans des pensionnats installés en différents endroits de l'Europe, éduqués en langue russe, dans un environnement que l'on voulait apaisé, où ils pouvaient retrouver les bases d'une vie dans la communauté russe. Pour l'anecdote, mais est-ce bien une anecdote quand il s'agit d'affronter les épreuves d'un exil toujours douloureux, on signalera l'existence d'une colonie russe installée dans la Tunisie du Protectorat à partir de 1922. La flotte de la mer Noire quitte en effet Sébastopol en novembre, composée de 150 unités avec 150000 personnes environ, militaires et civils. Après de difficiles péripéties pour trouver un accueil, 35 unités avec plus de 6000 personnes à leur bord eurent l'autorisation des autorités françaises d'accoster à Bizerte. Anastasia Manstein-Chirinsky, enfant en ce temps-là, raconta cette arrivée et cette installation à Bizerte. On en trouvera le récit dans un livre de souvenirs qu'elle publia à Tunis sous le titre La Dernière escale. Le siècle d'une exilée russe à Bizerte, Tunis, SudÉditions, 2004. On ouvrit une petite école sur l'un des navires où les enfants purent retrouver les bases d'une éducation et d'une formation dispensée en langue russe. Mais l'expérience ne dura pas longtemps. Les autorités françaises procédèrent à la dissolution de l'escadre. La plus grande partie des membres de cette communauté quittèrent la Tunisie pour se rendre en Europe. Seules un peu plus de 700 personnes restèrent en Tunisie. L'auteur poursuivit ses études dans les écoles de Bizerte, puis devint professeur de mathématiques dans cette même ville et y mourut en 2009.

2. On peut encore consulter, sur cette question, le mémoire de Louise Canette, Les enfants d'immigrés italiens dans les écoles françaises (1935-1955),université de Nantes http:// www.memoireonline.com/04/11/4444/m_Les-enfants-dimmigres-italiens-dans-les-ecolesfranaises-1935-19550.html

3. Lire sur ce sujet: Marie-Claude Blanc-Chaléard, « Les trois temps du bal musette ou la place des étrangers", in J.-L Robert et D. Tartakowsky (dir.), Paris le peuple, XVIII ${ }^{e}-\mathrm{XX}^{e}$ siècle, Paris, Publications de la Sorbonne, 1999, p. 77-90. 


\section{AUTEUR}

GÉRARD VIGNER

SIHFLES

g.vigner@noos.fr 